\title{
A preliminary study on translational kinetic energy absorption using coconut-fiber (Coir) sheets as a potential impact-worthy constituent in advanced aerospace material
}

\begin{abstract}
Known as "coir", the fibrous husk of the coconut fruit has potential for integration as a constituent in impact-resisting aerospace materials. As a preliminary study, kinetic energy absorption of this natural fiber is studied prior to further testing, for instance; a non-ballistic surface impacted at high velocity by a small mass is the equivalent mock-up to runway debris. The purpose of this study is to find the relationship between the thickness of the fiber with the kinetic energy absorption. Fabricated fiber panels measuring $10 \times 10 \times \mathrm{t} \mathrm{cm}$ with various thickness are subjected to mild steel projectiles launched by a light gas gun at a constant pressure. The velocity of the projectiles is set to be consistent with the velocity range of typical transport-category aircraft. The impact response of the panels aids in predicting the required amendments where plies of coir sheets are increased to which perforation is impossible. The relationship established from the experimental results is then used to predict the amount of layers required for total translational kinetic energy absorption.
\end{abstract}

Keyword: Coconut; Coir; Fiber; Energy; Impact; Kinetic; Translational 